\title{
Spectroscopic observations of the short-period, RS CVn-type star SV Cam ${ }^{\star}$
}

\author{
D. P. Kjurkchieva ${ }^{1}$, D. V. Marchev ${ }^{1}$, and S. Zola ${ }^{2,3}$ \\ 1 Department of Physics, Shoumen University, 9700 Shoumen, Bulgaria \\ and Isaac Newton Institute of Chile, Bulgarian Branch \\ e-mail: d.kyurkchieva@shu-bg.net; e-mail: d.marchev@shu-bg.net \\ 2 Astronomical Observatory, Jagiellonian University, ul. Orla 171, 30-244 Cracow, Poland \\ 3 Mt. Suhora Observatory of the Pedagogical University, ul. Podchorazych 2, 30-094 Cracow, Poland
}

Received 25 September 2001 / Accepted 12 February 2002

\begin{abstract}
Spectroscopic observations of the eclipsing, short-period RS CVn-star SV Cam in the range 6500-6700 $\AA$, covering the whole orbital period are presented. The absorption profiles of the lines $\mathrm{H}_{\alpha}$ and FeI 6678 are doubled out of the eclipses. The relative contribution of the secondary star is stronger in the FeI 6678 line than in $\mathrm{H}_{\alpha}$. We determined $K_{1}=123.1 \pm 1.2 \mathrm{~km} \mathrm{~s}^{-1}$ and $K_{2}=207.6 \pm 1.8 \mathrm{~km} \mathrm{~s}^{-1}$ by measurements of the Doppler shifts of these lines. The resulting masses of the components are: $M_{1}=1.47 \pm 0.06 M_{\odot}$ and $M_{2}=0.87 \pm 0.06 M_{\odot}$. From the radial velocity solution and photometrically obtained relative stellar radii we determined the absolute radii of the components: $R_{1}=1.38 \pm 0.05 R_{\odot}$ and $R_{2}=0.94 \pm 0.06 R_{\odot}$. The equatorial velocities $V_{\mathrm{eq}}^{1}=116 \mathrm{~km} \mathrm{~s}^{-1}$ and $V_{\mathrm{eq}}^{2}=79 \mathrm{~km} \mathrm{~s}^{-1}$ corresponding to the measured rotational broadenings of the investigated lines coincide with the velocities calculated from the obtained stellar radii. The phase behavior of both $\mathrm{H}_{\alpha}$ and FeI 6678 profiles of the primary star shows the presence of two cool spots with maximum visibilities at phases 0.27 and 0.86 . The analysis of the two lines in the middle of the primary eclipse allows us to illustrate the excess of the $\mathrm{H}_{\alpha}$ emission of the secondary star. The parameters of the stellar components of SV Cam derived from our spectroscopy and photometry are in very good agreement. The final result of our investigation is that there are three sources of activity in SV Cam: local active regions (photospheric spots) on its primary component, enhanced chromospheric emission from the secondary star and some additional emission originating from circumstellar gas.
\end{abstract}

Key words. stars: activity - binaries: eclipsing - binaries: spectroscopic - stars: chromospheres stars: individual: SV Cam - stars: starspots

\section{Introduction}

Stars which are forced into rapid rotation through tidal interaction in binary systems exhibit high levels of activity. A large number of observations have demonstrated the fundamental importance of stellar rotation for the generated activity level. Studies which relate the characteristics of star activity to basic star parameters are a natural way to test dynamo theories.

The strong photospheric, chromospheric, transition region and coronal activity in RS CVn stars are generally attributed to the deep convection zone and the fast rotation that drives the dynamo mechanism. Enhanced emission cores in the CaII $\mathrm{H}$ and $\mathrm{K}$ as well as the $\mathrm{H}_{\alpha}$ lines are the primary optical indicators of the chromospheric

Send offprint requests to: S. Zola, e-mail: zola@astro1.as.wsp.krakow.pl

* Research supported in part by grants from the Queen Jadwiga Foundation of the Jagiellonian University and project 2/2001 of Shoumen University. activity (Fernandez-Figueroa et al. 1994). Analysis of the spectra at different orbital phases allows us to establish which star is the source of the emission. If the activity of RS CVn stars is linked to the stellar dynamo then the short-period RS CVn stars have a proportionally larger supply of rotational energy to be converted into magnetic energy.

The eclipsing binary star SV Cam $(\mathrm{G} 2-3 \mathrm{~V}+\mathrm{K} 4 \mathrm{~V})$ belongs to the subgroup of short-period RS CVn systems and it is one of the closest binaries among them $\left(P_{\text {orb }}=\right.$ 0.6 days) with an orbital inclination $i \geq 80^{\circ}$. The star shows remarkable changes out of the eclipses. Variations of the light curve shape by as much as $0.1 \mathrm{mag}$ on time scales as short as one month have been observed. Extensive observations from 1973 until 1980 led Patkos (1982a,b) to the conclusion that cool spots on the surface of the primary component and sporadic gas streams are present in the system. Hempelmann et al. (1997) found evidence for chromospheric activity of the secondary star of SV Cam by an analysis of the strength of the $\mathrm{H}_{\alpha}$ absorption line 
during the primary and secondary eclipses. SV Cam does not show all of the signatures of RS CVn-type activity: there is no apparent $\mathrm{H}_{\alpha}$ emission, neither radio emission nor flare activity have been reported.

Results from X-ray observations of SV Cam are contradictory. Agrawal et al. (1980) and Welty \& Ramsey (1995) reported high X-ray luminosity and surprisingly large X-ray surface flux compared to that observed in semi-detached, Algol-type systems (Singh et al. 1980). On the other hand, Hempelmann et al. (1997) found the total X-ray output from SV Cam to be on a level typical of single Main Sequence stars, i.e. the observed coronal activity of this system is not enhanced compared to single stars. In semi-detached systems the mass transfer through the inner Lagrangian point is responsible for the higher X-ray flux. In case of SV Cas, however, due to its low Roche lobe filling factor this explanation is not valid. Hempelmann et al. (1997) modelled the X-ray light curve of SV Cam with two localized coronal emission regions rather than by global emission coming either from a homogeneously radiating stellar corona or from a common envelope surrounding the two stars. Although SV Cam is photometrically a well-studied star, only the primary's radial velocity curve was known until Pojmanski (1998), thus making any inferences from analysis of the data unreliable.

In order to search for a presence of the secondary star's lines in the spectrum of SV Cam and to investigate the phase behavior of the $\mathrm{H}_{\alpha}$ line which is a spectroscopic indicator of stellar chromospheric activity (Zarro \& Rogers 1983; Herbig 1985; Frasca \& Catalano 1994; Strassmeier et al. 1990), we decided to observe SV Cam in the spectral range around this line. An additional reason for undertaking these spectral observations was to compare the derived star parameters with those obtained from the light curve modeling. The multicolor light curve of SV Cam in 1997 was modeled with two circular cool spots having an angular size of $20^{\circ}$ located in the middle latitudes above and below the star equator, on opposite hemispheres of the primary star surface (Kjurkchieva et al. 2000a, hereafter Paper I).

Spectroscopic observations are described in the next section. In Sect. 3 we present the new radial velocity solution. The evidence of the enhanced $\mathrm{H}_{\alpha}$ emission of the secondary star is shown by an analysis of the line profiles in the middle of the primary eclipse. In Sect. 4 we discuss our spectral data in the framework of the dynamo theory of stellar activity and show a good agreement of the values of the parameters of stars determined both from our spectral and photometric data.

\section{Observations}

SV Cam was observed in the spectral range $6500-6700 \AA$ with resolution $0.19 \AA /$ pixel on 3 consecutive nights from November 30 to Dec. 2, 1999. We used a CCD camera mounted on the Coude spectrograph (grating $B \& L 632 / 14.7^{\circ}$ ) of the $2 \mathrm{~m}$ telescope operated by the National Astronomical Observatory in Rozhen (Bulgaria).
The seeing during the observations did not exceed 2 arcsec $(F W H M)$. The exposure time was $20 \mathrm{~min}$. The bias frames and flat-field integrations were obtained at the beginning and end of each night. All stellar integrations were alternated with Fe comparison source exposures. The data were processed in a standard way using the PCIPS (Smirnov et al. 1992) and Rewia (Borkowski 1988) software packages. The $S / N$ ratio was around $120-160$. The spectra were reduced by bias substraction, flat-field division and wavelength calibration. The normalization of the continuum was made to the whole line-free spectral range.

Apart from the $\mathrm{H}_{\alpha}$ line, in the range 6500-6700 $\AA$ there are several weaker Fe lines, whose radial velocities change with the orbital period of SV Cam. However, only the $\mathrm{H}_{\alpha}$ and FeI $6678 \AA$ lines show apparent double profiles out of the eclipses. For instance, the FeI 6593 line is stronger than FeI 6678 but it is blended with surrounding lines whose contribution is difficult to separate. An appropriate Fourier noise filter was applied to the weaker FeI 6678 line in order to remove the high-frequency noise. This procedure causes some smoothing of the profiles without loss of their important details (Gray 1992).

The data were phased using the ephemeris of Kreiner (2001):

$J H D(\operatorname{MinI})=2426949.3881+0.59307167 * E$.

\section{Analysis of the data}

\subsection{Radial velocity curve}

The measurements of the lines of the short-period RS CVn systems are difficult for several reasons. First, the lines arising from each component are rotationally broadened and blended with the surrounding metal lines of itself and the companion star. Second, the profiles are distorted by the presence of emission features. The absorption $\mathrm{H}_{\alpha}$ and FeI 6678 lines show double profiles outside the eclipses. Although the FeI 6678 line is significantly weaker than $\mathrm{H}_{\alpha}$ its doubling is better pronounced.

In order to determine the radial velocity of the lines we tried both gaussian and 6th-order polynomial fits. It turned out that the obtained values agree to within $0.045 \AA\left(2 \mathrm{~km} \mathrm{~s}^{-1}\right)$ (Fig. 1). The measured values of the radial velocities together with their error are given in Table 1 and shown in Fig. 2 where the values of the FeI line are phase shifted by 0.01 in order to avoid superposing of the errors bars. The radial velocities for the two lines were derived by fitting a sinusoid curve to the data (shown in Fig. 2) and the result of the least square fit is: $K_{1}=123.1 \pm 1.2 \mathrm{~km} \mathrm{~s}^{-1}, K_{2}=207.6 \pm 1.8 \mathrm{~km} \mathrm{~s}^{-1}$ and $\gamma=8.1 \pm 1.9 \mathrm{~km} \mathrm{~s}^{-1}$. It should be noted that our $\gamma$ value may not reflect the genuine systemic velocity since we did not observe the standard stars.

Our $K_{1}$ value is almost the same as that determined by Rainger et al. (1991) (122.3 $\left.\mathrm{km} \mathrm{s}^{-1}\right)$ and that of Hiltner (1953) $\left(123 \mathrm{kms}^{-1}\right)$ but it is slightly bigger than that of Hempelmann et al. (1997) $\left(117 \mathrm{~km} \mathrm{~s}^{-1}\right)$ and that of Pojmanski (1998) (118.3 $\left.\mathrm{km} \mathrm{s}^{-1}\right)$. Our value of $K_{2}$ is close 
Table 1. Radial velocities of the $\mathrm{H}_{\alpha}$ and FeI 6678 lines in $\mathrm{km} \mathrm{s}^{-1}$.

\begin{tabular}{|c|c|c|c|c|c|c|c|c|}
\hline Phase & $R R V_{1}\left(\mathrm{H}_{\alpha}\right)$ & $\overline{\Delta R V_{1}\left(\mathrm{H}_{\alpha}\right)}$ & $\bar{R} R V_{2}\left(\mathrm{H}_{\alpha}\right)$ & $\bar{\Delta} \Delta R V_{2}\left(\mathrm{H}_{\alpha}\right)$ & $\bar{R} R V_{1}(\mathrm{FeI})$ & $\bar{\Delta} \Delta R V_{1}(\mathrm{FeI})$ & $\bar{R} R V_{2}(\mathrm{FeI})$ & $\overline{\Delta \Delta R V_{2}(\mathrm{FeI})}$ \\
\hline 0.105 & -60.4 & 7.60 & & & -69.6 & 24.78 & 121.3 & 11.70 \\
\hline 0.130 & -74.0 & 10.60 & & & -73.0 & 19.13 & 134.0 & 15.20 \\
\hline 0.153 & -90.0 & 3.42 & & & -96.6 & 23.04 & 164.0 & 28.00 \\
\hline 0.177 & -94.6 & 6.09 & & & -105.6 & 20.86 & 191.0 & 28.00 \\
\hline 0.200 & -96.9 & 6.66 & & & -114.5 & 20.86 & 195.0 & 25.80 \\
\hline 0.225 & -99.2 & 8.53 & & & -116.8 & 26.95 & & \\
\hline 0.250 & -101.5 & 11.26 & 205.0 & 27.50 & -119.0 & 28.69 & 218.0 & 50.40 \\
\hline 0.270 & -106.0 & 10.66 & & & -123.5 & 23.04 & 195.4 & 21.06 \\
\hline 0.295 & -103.7 & 9.13 & 200.0 & 25.00 & -105.6 & 19.13 & 183.0 & 18.66 \\
\hline 0.320 & -101.5 & 7.93 & 195.0 & 27.40 & -105.6 & 30.43 & 173.0 & 33.60 \\
\hline 0.590 & 81.4 & 10.93 & & & 62.9 & 26.52 & -123.0 & 30.13 \\
\hline 0.613 & 92.8 & 8.00 & & & 78.6 & 24.78 & -132.5 & 26.66 \\
\hline 0.637 & 104.2 & 6.06 & & & 96.5 & 11.73 & & \\
\hline 0.660 & 111.1 & 5.80 & & & 110.0 & 22.60 & & \\
\hline 0.668 & 118.0 & 4.93 & -193.0 & 22.00 & 119.0 & 27.40 & & \\
\hline 0.700 & 122.5 & 4.80 & -199.7 & 17.80 & 128.0 & 28.20 & -195.4 & 49.06 \\
\hline 0.720 & 145.3 & 4.92 & & & 134.7 & 20.80 & & \\
\hline 0.740 & 151.0 & 6.26 & & & 137.0 & 27.39 & -213.9 & 49.06 \\
\hline 0.766 & 145.3 & 6.27 & -201.0 & 20.30 & 130.0 & 23.40 & -213.0 & 41.86 \\
\hline 0.790 & 140.3 & 6.26 & -197.0 & 21.65 & 123.0 & 23.47 & -204.0 & 45.33 \\
\hline 0.814 & 137.0 & 5.93 & -193.0 & 24.20 & 114.0 & 27.40 & -198.0 & 30.93 \\
\hline 0.840 & & & & & 105.0 & 29.13 & & \\
\hline 0.860 & & & & & 96.5 & 21.30 & -186.0 & 19.20 \\
\hline
\end{tabular}

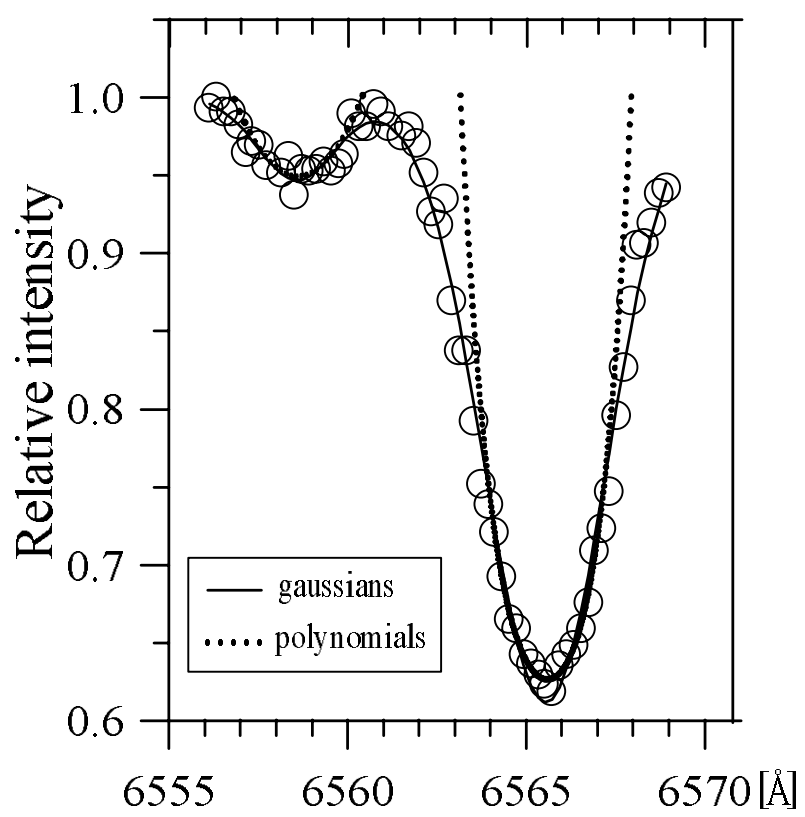

Fig. 1. An illustration of the fitting procedure.

to the only value known so far, published by Pojmanski $\left(211.5 \mathrm{~km} \mathrm{~s}^{-1}\right)$ and obtained from the analysis of near IR CaII spectral lines.

Assuming the photometrically determined value of $i=$ $80^{\circ}$ (Hilditch et al. 1979; Kjurkchieva et al. 2000a) and using our values for $K_{1}$ and $K_{2}$ the resulting values of the

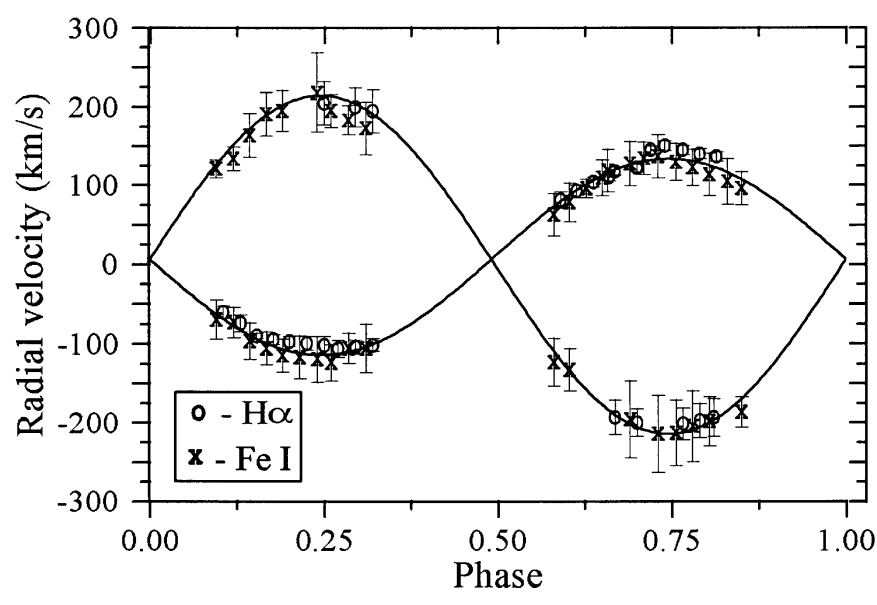

Fig. 2. Radial velocity curves of SV Cam.

mass ratio and masses of the components are $q=0.593 \pm$ $0.011, M_{1}=1.47 \pm 0.06 M_{\odot}$ and $M_{2}=0.87 \pm 0.06 M_{\odot}$.

On the basis of our radial velocity solution and photometrically obtained fractional radii $r_{1}=0.35$ and $r_{2}=$ 0.24 (Paper I), we determined the absolute star's radii $R_{1}=1.38 \pm 0.05 R_{\odot}$ and $R_{2}=0.94 \pm 0.06 R_{\odot}$. It should be noted that the values of $r_{1}$ and $r_{2}$ determined by different authors lie in the ranges 0.32-0.4 and 0.19-0.25 (Budding \& Zeilik 1987; Zeilik et al. 1988; Patkos \& Hempelmann 1994; Djurasevic 1998; Heckert et al. 1998). The radii of the components of SV Cam corresponding to their masses calculated from the mass-radius relation for MS stars are $R_{1}=1.32 R_{\odot}$ and $R_{2}=0.91 R_{\odot}$ for the primary and 


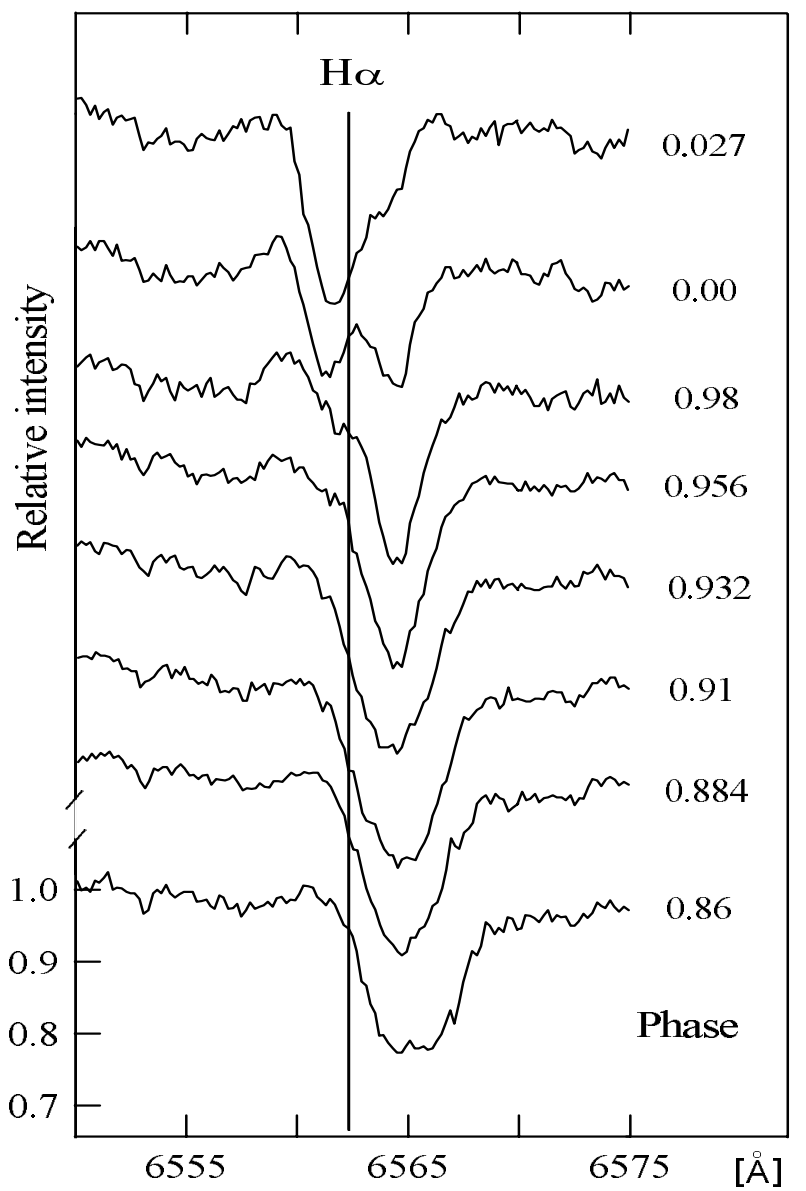

Fig. 3. $\mathrm{H}_{\alpha}$ profiles of SV Cam from Nov. 30 .

secondary, respectively. This means that the masses and the radii of the two components almost obey the massradius relation for MS stars in spite of their gravity distortions.

We determined the rotational broadenings of the two profiles of the investigated lines by measurements of the widths at the continuum level of the 6 th order polynomial fit (Fig. 1). The mean values of the full width of the broadened profiles are almost the same for the $\mathrm{H}_{\alpha}$ and FeI 6678 lines: $\Delta \lambda_{1}^{\text {rot }}=5 \pm 0.2 \AA$ for the primary profiles and $\Delta \lambda_{2}^{\text {rot }}=4 \pm 0.3 \AA$ for the secondary ones. The corresponding equatorial velocities to these rotational broadenings $\left(2 V_{\mathrm{eq}} \sin i=c \Delta \lambda_{\mathrm{rot}} / \lambda\right)$ are $V_{\mathrm{eq}}^{1}=116 \pm 9 \mathrm{~km} \mathrm{~s}^{-1}$ and $V_{\mathrm{eq}}^{2}=79 \pm 14 \mathrm{~km} \mathrm{~s}^{-1}$. For comparison, assuming synchroneous rotation of the components and the obtained stars' radii the calculated equatorial velocities of the stars $\left(V_{\text {eq }}=2 \pi R / P_{\text {rot }}\right)$ are $V_{\text {eq }}^{1}=117.7 \mathrm{~km} \mathrm{~s}^{-1}$ and $V_{\mathrm{eq}}^{2}=80.7 \mathrm{~km} \mathrm{~s}^{-1}$. Hence, in spite of the fact that the spectral lines are distorted by different effects their widths agree with the expected rotational broadenings.

\subsection{Phase behavior of the profiles}

The normalized $\mathrm{H}_{\alpha}$ and FeI 6678 profiles are shown in Figs. 3-12 together with the corresponding orbital phases.

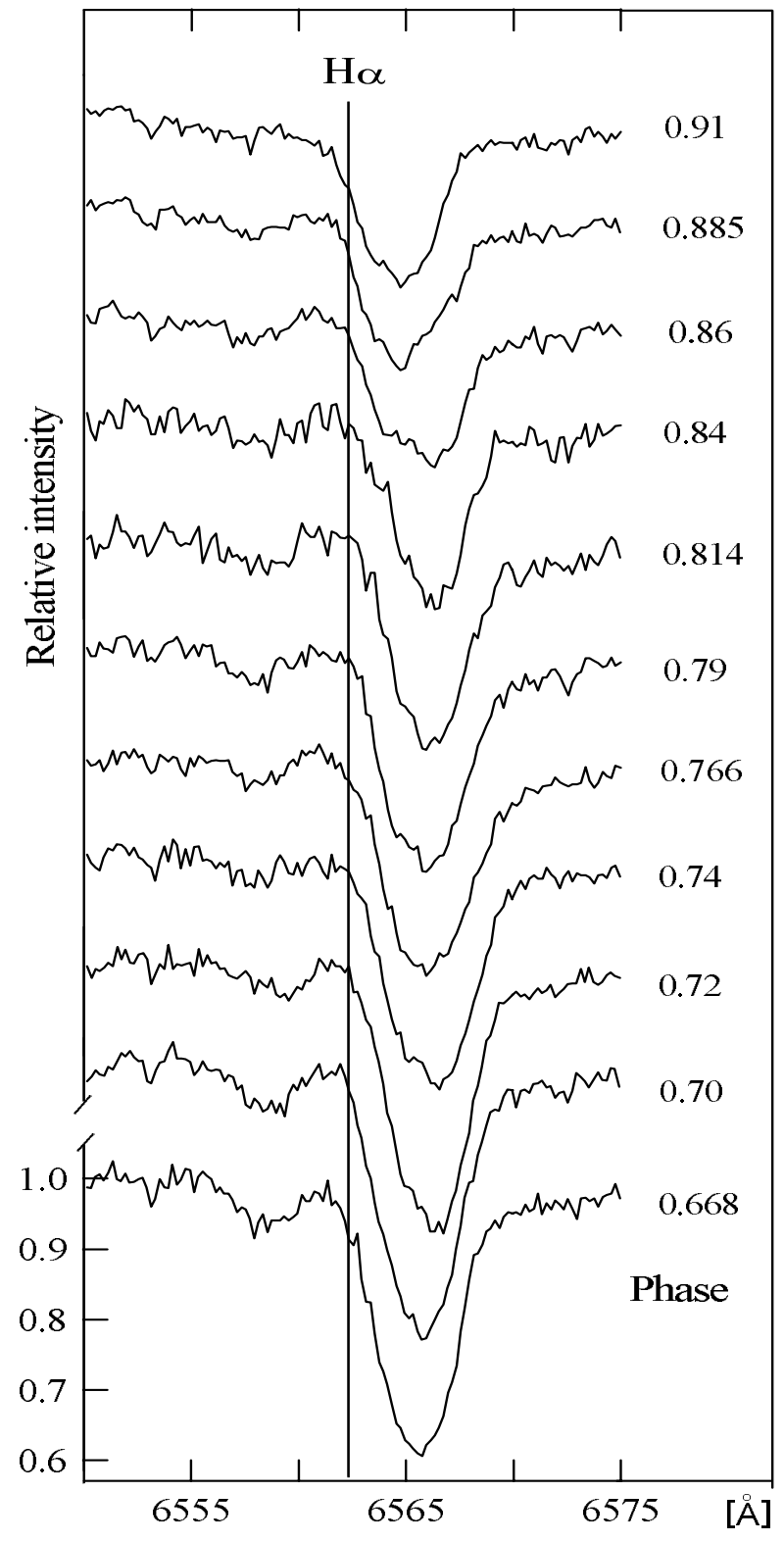

Fig. 4. $\mathrm{H}_{\alpha}$ profiles of SV Cam from Dec. 1.

We established the following pecularities in the phase behavior of the $\mathrm{H}_{\alpha}$ profile of the primary star:

(1) The profile has a central pseudo-emission bump in the middle of the primary eclipse. We attribute this distortion to the obscuration of the primary star by the cooler secondary that causes masking of a part of the primary's absorption line. The width of the central bump is $3.4 \AA$ and corresponds precisely to the secondary star's radius (see the previous section).

(2) The profile is deeper around the second quadrature than around the first one. The shape of the $\mathrm{H}_{\alpha}$ profile is symmetrical at the second quadrature and distorted at the first quadrature.

(3) Some emission feature at the end of the left wing of the profile appears at phase range $0.95-0.98$ that repeats on two dates (Figs. 3 and 5). One can also note a similar, weaker emission feature almost half orbital cycle 


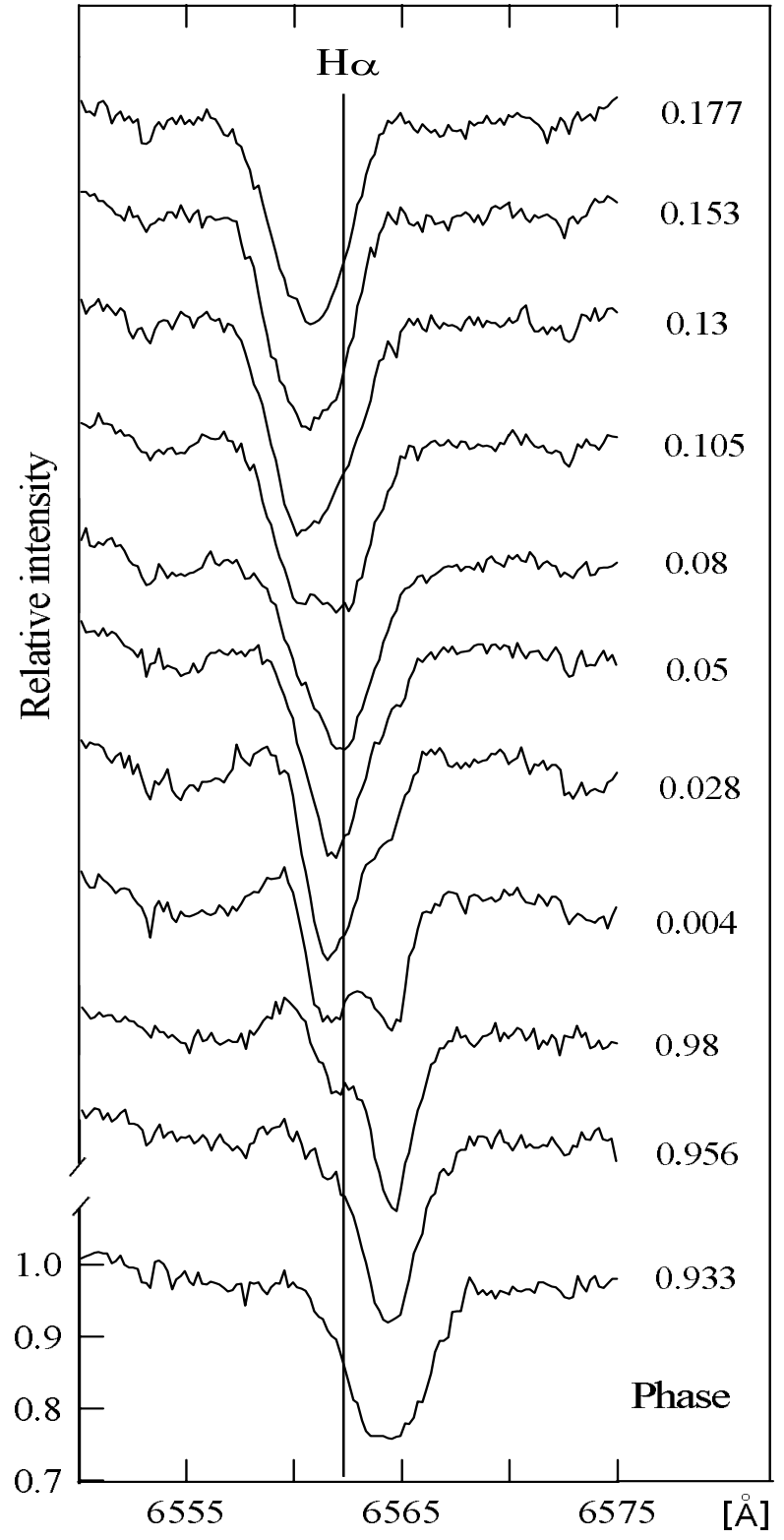

Fig. 5. $\mathrm{H}_{\alpha}$ profiles of SV Cam from Dec. 1, continued.

later (in the phase range $0.54-0.56$ ) at the end of the right wing. This feature may be due to the emission from circumstellar matter, perhaps ejected from the component(s) whose contribution is most apparent in the spectra around the eclipses. The presence of circumstellar matter in SV Cam was suspected a long time ago on the basis of its optical light curves (Patkos 1982a,b) and the X-ray data (Hempelmann et al. 1997). Another reason for the emission feature can be the increased contribution of the trailing and leading part of the secondary star chromosphere in the corresponding phase ranges.

(4) The profile has a nearly flat core at phases 0.86 , 0.105 and 0.79 . The first two phases are almost symmetric relative to the primary eclipse. We found the same behavior in the $\mathrm{H}_{\alpha}$ line of the primary star in the short-period RS CVn-type star RT And (Kjurkchieva et al. 2001).

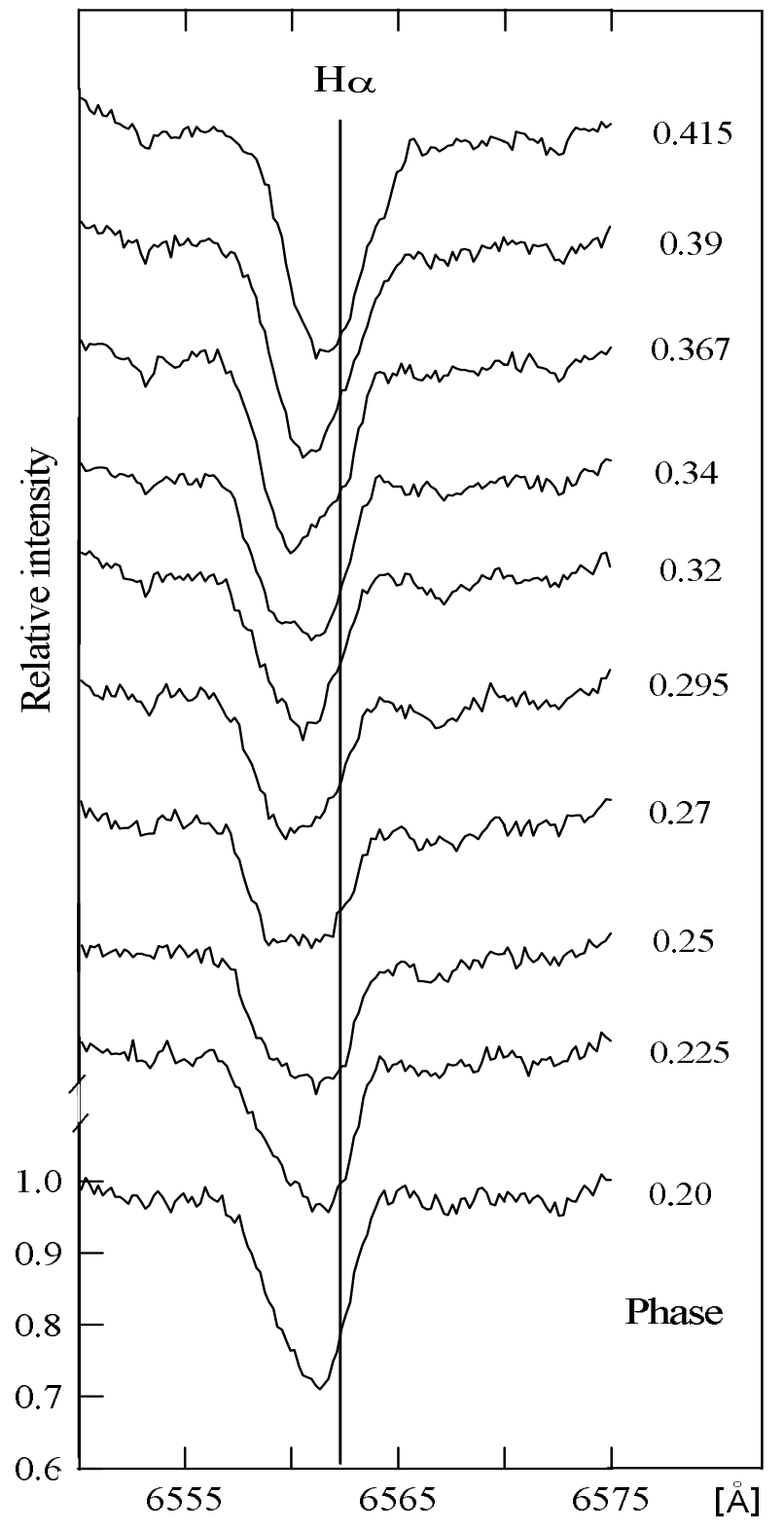

Fig. 6. $\mathrm{H}_{\alpha}$ profiles of SV Cam from Dec. 1, continued.

(5) The profile is distorted and filled-in in the phase ranges $0.2-0.34$ and $0.84-0.91$. The distortions of the profile are variable and seem to be caused by features that moves through the stellar disk during the above phases (see Figs. 4 and 6). They could be attributed, at least partially, to the effects of cool spots. The phases 0.27 and 0.86 of the biggest distortions (flat cores) of the $\mathrm{H}_{\alpha}$ profile almost coincide with phases of the maximum spot visibility determined by modeling of the SV Cam light curves (Paper I). It should be stressed out that the strong decrease of the profiles depths at the phase range 0.225 0.295 is difficult to explain only by spots with a reasonable size.

We established the following similarities in the phase behavior of the FeI 6678 and $\mathrm{H}_{\alpha}$ profiles of the primary star: (i) the depth of the FeI 6678 line is also greater around the second quadrature than around the first one 


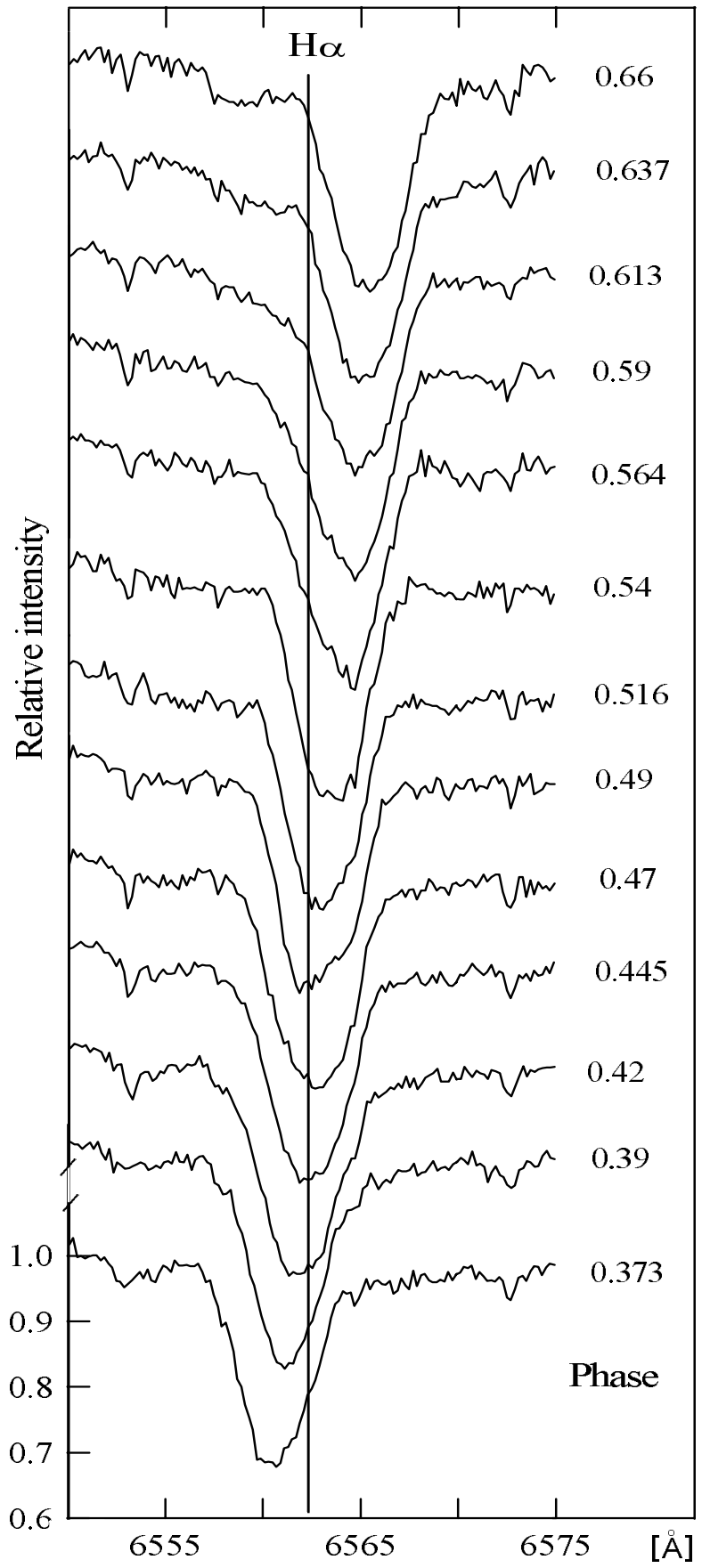

Fig. 7. $\mathrm{H}_{\alpha}$ profiles of SV Cam from Dec. 2.

but the difference between them is smaller for FeI 6678 ; (ii) there is also an emission feature at the end of the left wing of the profile at the phases 0.95-0.98; (iii) in the middle of the primary eclipse, the FeI 6678 profile also has a central pseudo-emission bump with the same width (about $3.4 \AA$ ), but its relative height is somewhat less than in $\mathrm{H}_{\alpha}$.

We found additional peculiarities of the FeI 6678 profile of the primary star:

(1) Apparent central emission features are visible at phases $0.25,0.84$ and 0.564 . The first two of them almost coincide with the phases of maximum visibility of the two

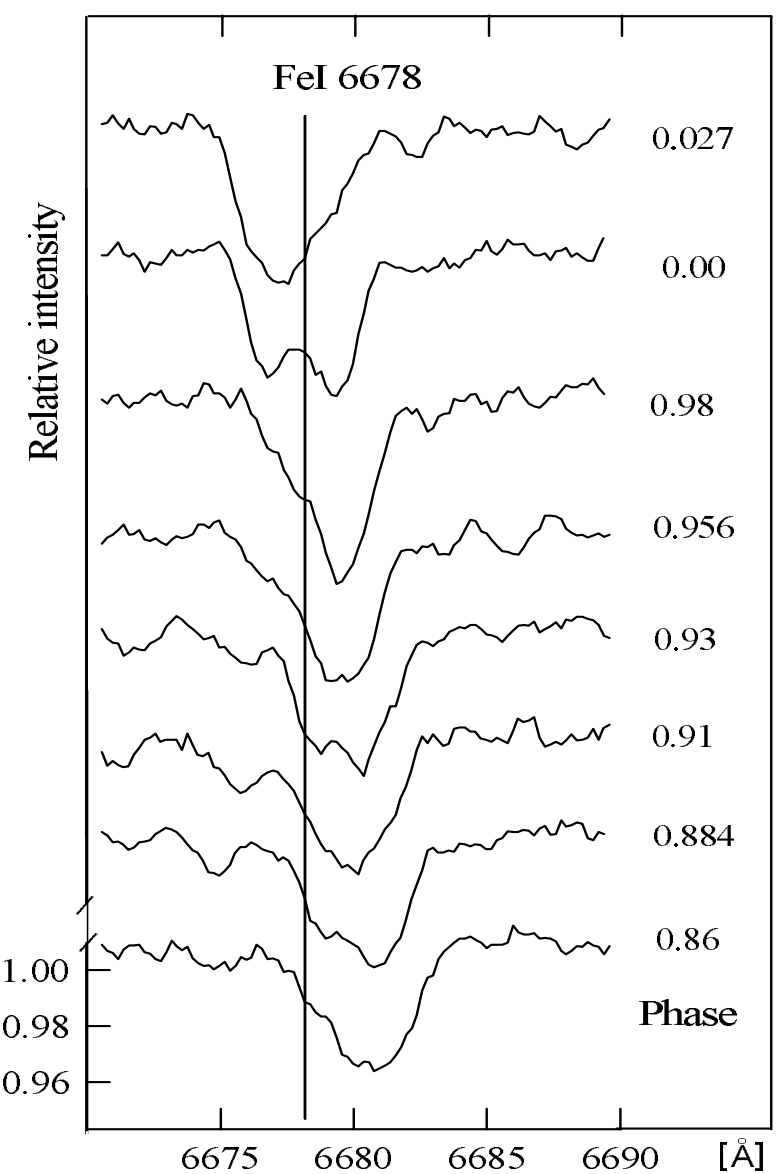

Fig. 8. FeI 6678 profiles of SV Cam from Nov. 30.

spots on the primary star reproducing the distortion light curve of SV Cam from 1997 (Paper I). We may calculate the angular size $\alpha^{\mathrm{sp}}$ of the spots from the equation (Kjurkchieva 1995):

$\frac{\Delta \lambda_{\mathrm{rot}}^{\mathrm{sp}}}{\Delta \lambda_{\mathrm{rot}}^{\mathrm{st}}}=\sin \alpha^{\mathrm{sp}}$

where $\Delta \lambda_{\text {rot }}^{\mathrm{sp}}$ and $\Delta \lambda_{\text {rot }}^{\mathrm{st}}$ are the rotational broadenings of the spot spectral feature and the star profile, respectively. From our spectra we measured: $\Delta \lambda_{\text {rot }}^{\mathrm{sp}}=1.6 \AA, \Delta \lambda_{\text {rot }}^{\mathrm{st}}=$ $5 \AA$ and obtained $\alpha^{\mathrm{sp}}=18^{\circ} .5$. This value is not far from the angular size of $20^{\circ}$ of the spots determined from the modeling of the light curve of SV Cam (Paper I);

(2) A strong increase of the depth of the profile is seen at phase 0.98 that repeats on two dates.

Consequently, the phase behavior of the $\mathrm{H}_{\alpha}$ and FeI 6678 profiles show the presence of two cool spots on the primary star of SV Cam. The Doppler image of SV Cam obtained by Hempelmann et al. (1997) also shows a distinct spot on the primary star whose location coincides with the spot found by modeling of the optical light curve.

Our observations out of the eclipses show a weak $\mathrm{H}_{\alpha}$ absorption profile from the secondary star that is deeper around the second quadrature than around the first one similarly to the behavior of the $\mathrm{H}_{\alpha}$ line of the primary star. For comparison, the $\mathrm{H}_{\alpha}$ profiles obtained by Hempelmann et al. (1997) revealed no features that could be attributed 


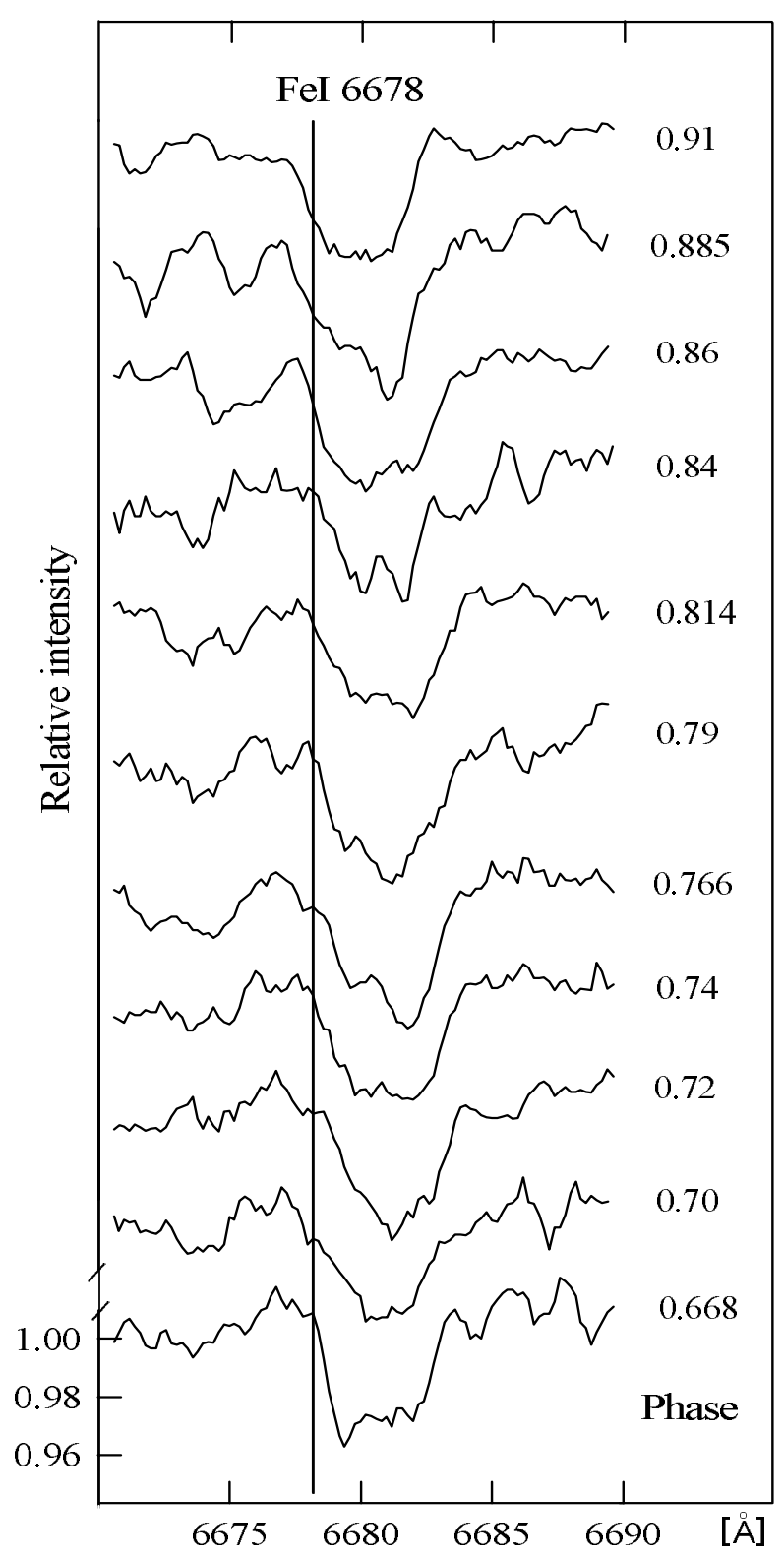

Fig. 9. FeI 6678 profiles of SV Cam from Dec. 1.

to the secondary star at the out-of-eclipse phases and they determined the contribution of the secondary star by an analysis of the single $\mathrm{H}_{\alpha}$ profile during both eclipses. Our observations of the FeI 6678 line outside the eclipses show also an absorption line from the secondary star. This line is shallower at quadratures than in the phases around them.

The central intensities $\left(R_{\mathrm{c}}=1-I_{\text {line }} / I_{\text {cont }}\right)$ of the primary's and secondary's $H_{\alpha}$ profiles change during the orbital cycle in the ranges $0.2-0.38$ and $0.02-0.07$ while intensities for the FeI 6678 line vary in the ranges $0.015-0.04$ and $0.005-0.01$.

\subsection{Emission from the stellar components}

Because we have no observations of standard, nonactive stars we cannot apply the widespread method of substraction of comparison spectrum from that of the

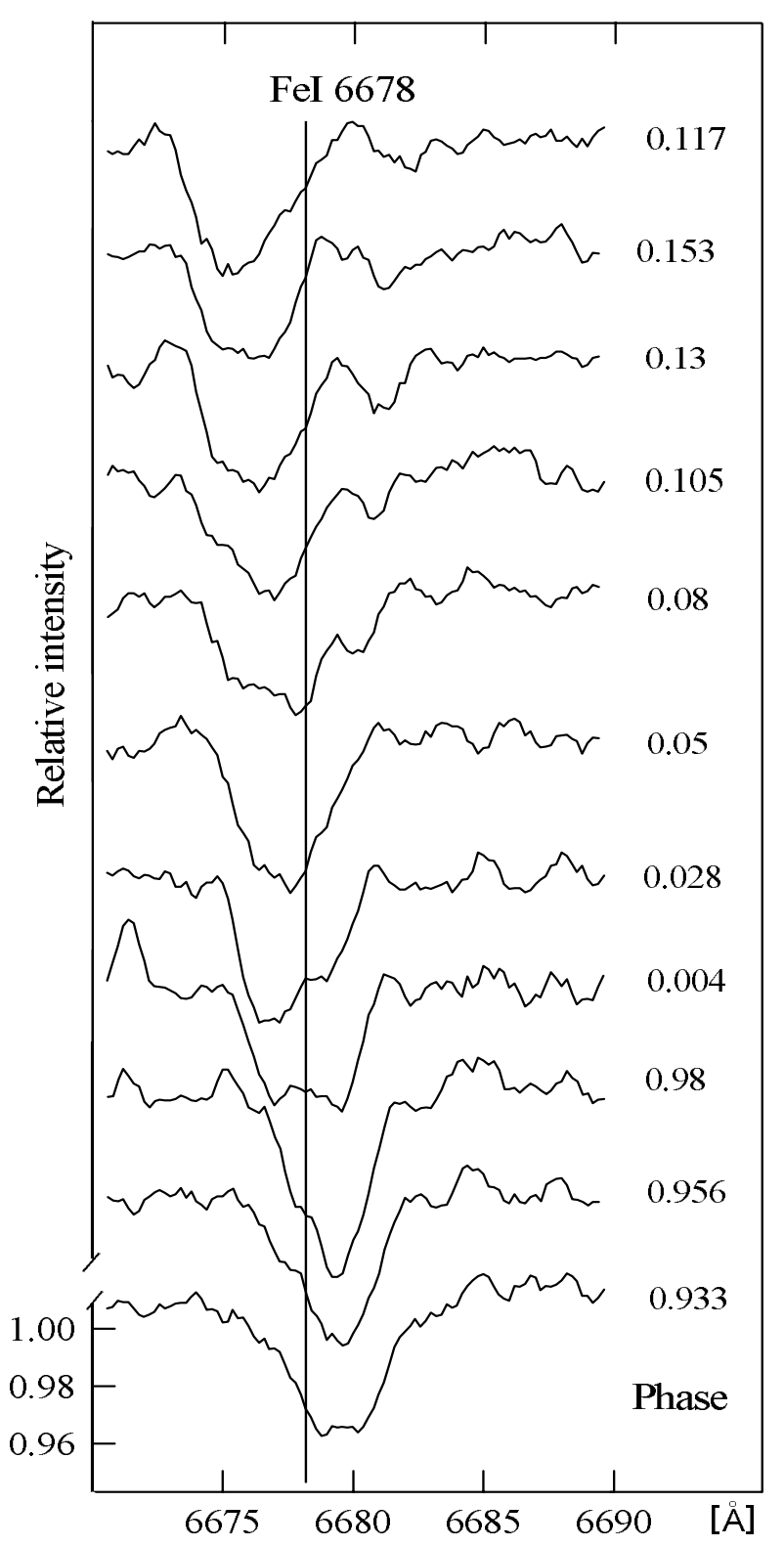

Fig. 10. FeI 6678 profiles of SV Cam from Dec. 1, continued.

active star (Strassmeier et al. 1990; Frasca \& Catalano 1994; Pojmanski 1998; Frasca et al. 2000, etc.). However, in order to obtain some information about the emission from the stellar components of SV Cam we analyzed the profiles at the primary eclipse. The line profile in the middle of this eclipse (adopting $\sin i \simeq 1$ ) is described roughly by the expressions:

$I(x)=2 I_{1} \int_{R_{2}}^{R_{1}} \cos \theta_{1} \mathrm{~d} z+2 I_{2} \int_{0}^{R_{2}} \cos \theta_{2} \mathrm{~d} z$

for $-R_{2}<x<R_{2}$ and

$I(x)=2 I_{1} \int_{0}^{R_{1}} \cos \theta_{1}$

for $-R_{1}<x<-R_{2}$ and $R_{2}<x<R_{1}$ where

$\cos \theta_{i}=\frac{\sqrt{R_{i}^{2}-x^{2}-z^{2}}}{R_{i}}$ 


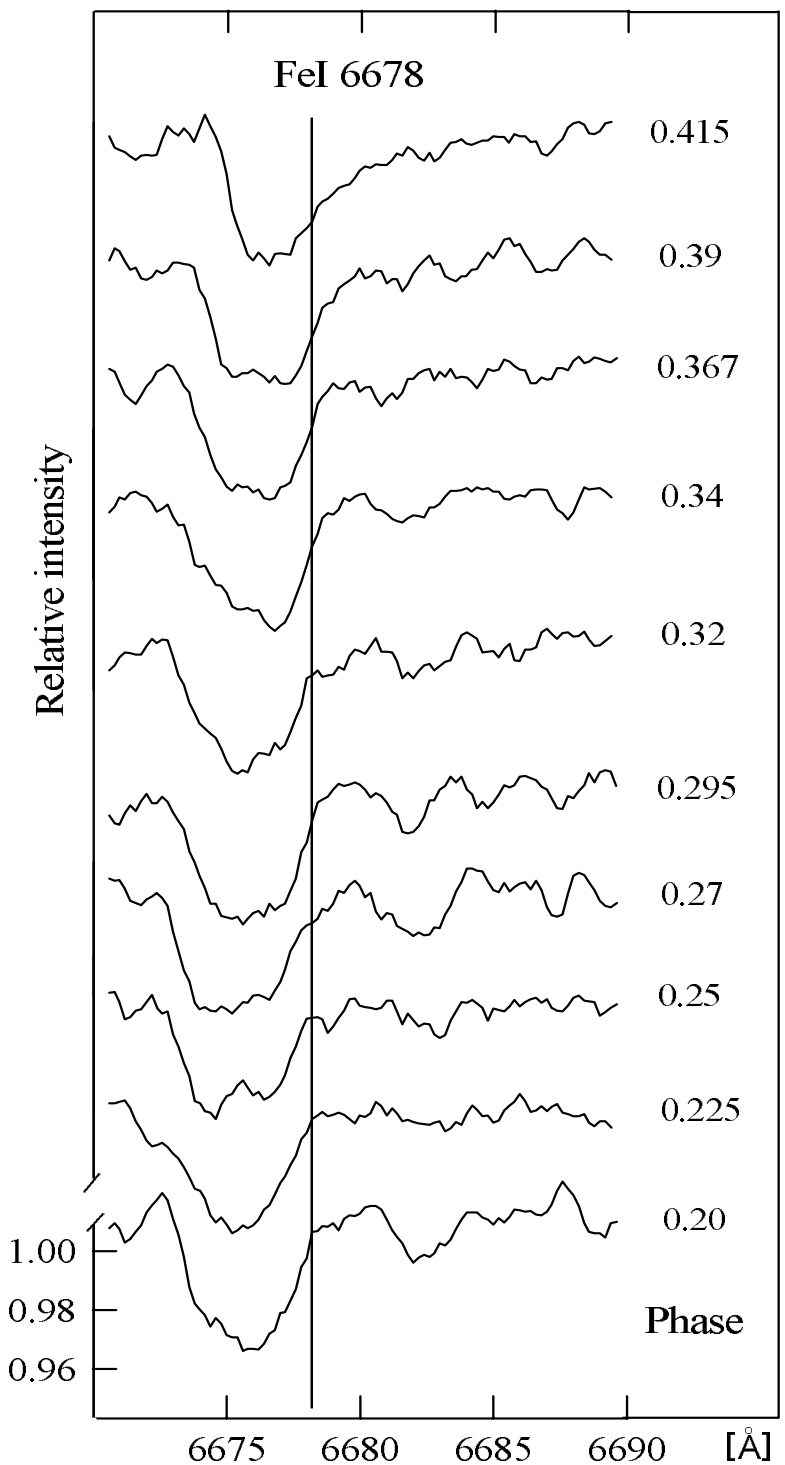

Fig. 11. FeI 6678 profiles of SV Cam from Dec. 1, continued.

$x=\left(1-\lambda / \lambda_{0}\right) c R_{i} / V_{\mathrm{eq}}^{i}$,

$I_{1}$ and $I_{2}$ are the surface intensities (they are positive for emission lines and negative for absorption lines) of the primary and secondary star at the wavelength of the line considered.

The expressions (3) and (4) describe the line profile with two-wave or $W$-like shape, i.e. a line profile with a central bump. The main parameters of this profile are:

(a) width of the bump $w_{\mathrm{c}}=2 \lambda_{0} V_{\mathrm{eq}}^{2} / c$;

(b) width of the whole profile $w=2 \lambda_{0} V_{\mathrm{eq}}^{1} / c$;

(c) depth of the center of the bump

$d_{\mathrm{b}}(0)=\frac{\pi I_{2} R_{2}}{2}+\frac{\pi I_{1} R_{1}}{2}-I_{1} R_{1}(k \omega+\arccos \omega)$

where $k=R_{2} / R_{1}$ and $\omega=\sqrt{1-k^{2}}$;

(d) depths of the ends of the bump

$d_{\mathrm{b}}(e)=I_{1} R_{1} \omega^{2} \pi / 2$.

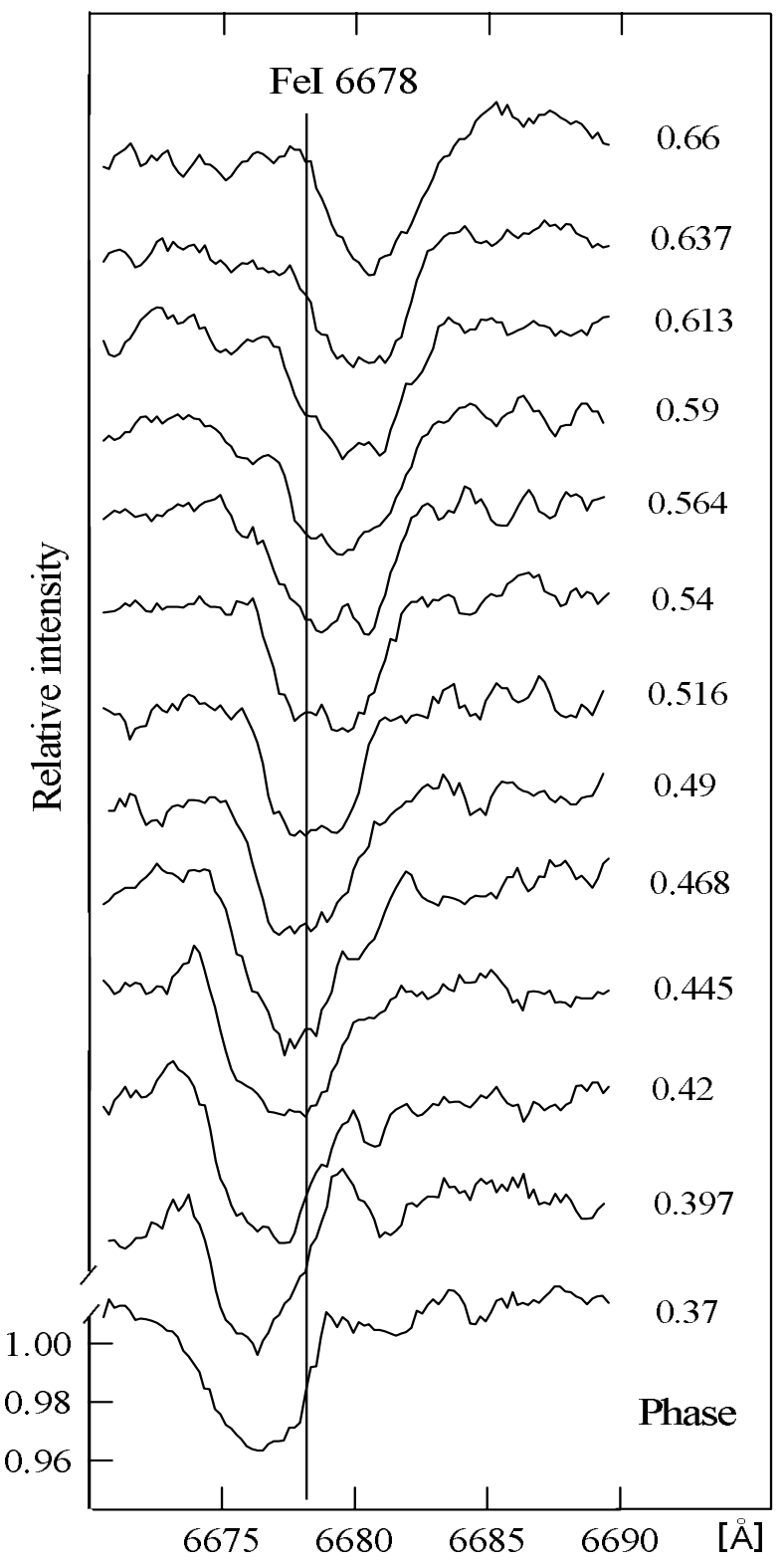

Fig. 12. FeI 6678 profiles of SV Cam from Dec. 2.

The depths of the centers of the primary's and secondary's profiles are respectively:

$d_{1}(0)=2 I_{1} R_{1} / \pi$

$d_{2}(0)=2 I_{2} R_{2} / \pi$.

From the above equations one can obtain the following simple relations:

$\frac{d_{\mathrm{b}}(e)}{d_{1}(0)}=\omega^{2}$

$\frac{d_{\mathrm{b}}(0)}{d_{1}(0)}=1+\frac{d_{2}(0)}{d_{1}(0)}-\frac{2(\omega \sqrt{k}+\arccos \omega)}{\pi}$.

The variability of the lines of the primary star during the cycle rises a question at which phase one should measure the value of $d_{1}(0)$. Because the $\mathrm{H}_{\alpha}$ line has most undistorted and symmetrical profile at the phase 0.67 we 
Table 2. Measured parameters of the $\mathrm{H}_{\alpha}$ and the FeI 6678 lines.

\begin{tabular}{lllll}
\hline \hline Line & $d_{1}(0)$ & $d_{2}(0)$ & $d_{\mathrm{b}}(0)$ & $d_{\mathrm{b}}(\mathrm{e})$ \\
\hline $\mathrm{H}_{\alpha}$ & 0.380 & 0.060 & 0.120 & 0.175 \\
FeI 6678 & 0.085 & 0.012 & 0.034 & 0.048 \\
\hline
\end{tabular}

decided to measure its depth at this phase. In order to compare parameters of the two investigated lines we measured $d_{1}(0)$ of the FeI 6678 line at the same phase, fitting its distorted profile with a gaussian curve. The measured values of the above parameters for the $\mathrm{H}_{\alpha}$ and FeI 6678 profiles are presented in Table 2 .

For the $\mathrm{H}_{\alpha}$ profile the measured and calculated values of $d_{\mathrm{b}}(0) / d_{1}(0)$ are respectively 0.316 and 0.38 , i.e. the calculated value is about $20 \%$ bigger than the observed one. The measured and calculated values of $d_{\mathrm{b}}(\mathrm{e}) / d_{1}(0)$ are respectively 0.46 and 0.55 , i.e. the calculated value is also about $20 \%$ bigger than the observed one. Consequently, the observed bump of the $\mathrm{H}_{\alpha}$ profile in the middle of the primary eclipse is higher than one would expect. We attribute this to the $\mathrm{H}_{\alpha}$ emission from the secondary star. For the FeI 6678 line, the measured and calculated values of $d_{\mathrm{b}}(0) / d_{1}(0)$ are 0.4 and 0.36 , respectively. Therefore, the calculated value for this parameter is smaller than the measured one. The measured and calculated values of $d_{\mathrm{b}}(\mathrm{e}) / d_{1}(0), 0.55$ and 0.56 , respectively agree very well. Thus the calculated profile of FeI 6678 in the middle of the primary eclipse is slightly above the observed one. In this way, our spectral observations show an enhanced emission of the secondary star of SV Cam in the $\mathrm{H}_{\alpha}$ line but not in the FeI 6678 line. The result of our analysis supports the conclusion of Ozeren et al. (2001) about the $\mathrm{H}_{\alpha}$ emission excess from the secondary star of SV Cam obtained by spectral substraction technique as well as the more general conclusion of Frasca \& Catalano (1994) about the $\mathrm{H}_{\alpha}$ emission in late-type active binaries.

\section{Discussion}

Montes et al. (1995) found that systems with $\mathrm{H}_{\alpha}$ emission above the continuum are all cooler than $5000 \mathrm{~K}$. The temperature of the primary star of SV Cam is above this limit and there should be no emission excess expected from it. Our spectral observations show some weak $\mathrm{H}_{\alpha}$ emission from the primary star that is connected with its large spots. Rainger et al. (1991) obtained 8 spectra of SV Cam in $\mathrm{H}_{\alpha}$ (with $S / N=40$ ) that displayed an apparently undisturbed line without any signature of emission.

The analysis of our spectral and photometric data shows that the activity of the primary star is connected with its local surface structures (photospheric spots). Because of the small contribution of the secondary star to the total light (around 10\%), it is difficult to find evidence for its spots by photometry as well as by spectroscopy. That is why we do not exclude the presence of active regions on the secondary's surface distributed relatively uniformly and close to each other, in contrast to the large spots on the primary star. But the quality of our spectral data allows us to conclude only that the secondary star shows some $\mathrm{H}_{\alpha}$ emission excess.

Finally we would like to point out the good agreement between the values for parameters of stars determined by analysis of the spectral data (this paper) and the photometric data (Paper I):

(1) The photometric ratio of the star radii $k=0.685$ is precisely the same as that determined by the rotational broadenings of their spectral lines $k=0.68$.

(2) The obtained value of the mass of the primary star corresponds to a F5V star. This value is in good agreement with the color index $B-V=0.42$ obtained on the basis of our multicolor photometry (Paper I). The mass of the primary star is significantly greater than previously assumed $\left(1 M_{\odot}\right)$ based on its adopted spectral type (Budding \& Zeilik 1987; Rainger et al. 1991; Patkos \& Hempelmann 1994). The G2-3V classification of Hilditch et al. (1979) was based on photometric observations in the DDO system and in the ubvy Stromgren system and would not give a reliable mass value if the primary star's surface were covered with large cool spots or if there was circumstellar matter present in the system. Our determination of the primary star mass is very close to the value $\left(1.42 M_{\odot}\right)$ obtained by Pojmanski (1998) from the near IR spectroscopic observations. We conclude that according to both our photometric and spectroscopic data the spectral types of the SV Cam components are F5 and K4.

(3) It is worth noting that the photometric ratio of the luminosities of the two components in the $\mathrm{R}$ band (the range of the two investigated spectral lines) $l=0.136$ is close to the ratio of the fluxes in the FeI 6678 line (equal to the ratio $E W_{2} / E W_{1}$ ) of 0.143 while the ratio of the fluxes in the $\mathrm{H}_{\alpha}$ profiles (0.085) is quite smaller. This fact also confirms the $\mathrm{H}_{\alpha}$ emission excess from the secondary star.

\section{Conclusions}

The results from the analysis of our spectral data of SV Cam could be summarized as follows:

1. The $\mathrm{H}_{\alpha}$ and Fe I 6678 lines have double profiles out of the eclipses. We measured $K_{1}=123.1 \mathrm{kms}^{-1}$ and $K_{2}=207.6 \mathrm{~km} \mathrm{~s}^{-1}$ and obtained the mass ratio $q=0.593$ and masses of the components $M_{1}=$ $1.47 M_{\odot}$ and $M_{2}=0.87 M_{\odot}$.

2. We found the spectral type of the primary component of SV Cam, corresponding to its mass, to be F5V. That is almost one spectral class earlier than previously published. Our spectral type is in good agreement with the color index $B-V=0.42$, obtained on the basis of our multicolor photometry.

3. The determined masses and radii of the stellar components of SV Cam obey the mass-radius relation for MS stars.

4. The measured rotationalal broadenings of the investigated lines correspond to equatorial velocities $V_{\mathrm{eq}}^{1}=$ $116 \mathrm{~km} \mathrm{~s}^{-1}$ and $V_{\mathrm{eq}}^{2}=79 \mathrm{~km} \mathrm{~s}^{-1}$. They agree very well 
with the values calculated from the obtained radii. Our value of $V_{\text {eq }}$ for the primary star is bigger than that (105 $\left.\mathrm{km} \mathrm{s}^{-1}\right)$ measured by Huisong \& Xuefu (1987) and Pojmanski (1998) and close to the value of $117 \mathrm{~km} \mathrm{~s}^{-1}$ determined by Hempelmann et al. (1997).

5. The relatively low $S / N$ ratio of our spectral data does not satisfy the strong requirements of the Doppler imaging technique for surface mapping of stars. We were able to reach only some qualitative conclusions about the surface inhomogeneities of the stellar components and to compare them with the results from the light curve modeling. The analysis of the phase behavior of both $\mathrm{H}_{\alpha}$ and FeI 6678 profiles of the primary star showed presence of two cool spots their with maximum visibilities at phases 0.27 and 0.86 . In spite of the long time interval between our photometric and spectroscopic observations (more than 2 years), it is surprising that the moving spectral features causing the distortion of the line profiles of the primary star is phase correlated with the visibilities of the two cool spots on its surface.

6. We found a very good agreement between the global parameters of stars determined from spectroscopic and photometric observations.

7. The line profiles of SV Cam are considerably deeper at the second quadrature than at the first one. The same behavior of the $\mathrm{H}_{\alpha}$ profile of SV Cam was observed recently by Ozeren et al. (2001). The explanation of the absorption established in AB Dor, HK Aqu, SS Boo by cool material in their co-rotating atmospheres (Collier Cameron et al. 1990; Byrne et al. 1996; Hall et al. 1990) is not applicable to our case because the excess absorption for two other short-period RS CVn stars, namely, XY UMa (Kjurkchieva et al. 2000b) and RT And (Kjurkchieva et al. 2001) is visible also around the second quadrature. We have not any explanation for this "quadrature effect" at this time.

As the final result of our investigation, we conclude that there are three activity sources in SV Cam: local active regions (photospheric spots) on the primary star that are observable in photometry as well as in the $\mathrm{H}_{\alpha}$ and FeI 6678 lines; enhanced chromospheric emission from the secondary star detectable in the $\mathrm{H}_{\alpha}$ line; emission of the circumstellar gas which presence is suspected both in the photometric and spectral data. Probably the three appearances of activity are correlated with each other according to the theory of interacting magnetospheres in RS CVntype stars (Uchida \& Sakurai 1983).

Acknowledgements. The authors are grateful to the referee for very useful remarks which allowed us to significantly improve this work.

\section{References}

Agraval, P., Riegler, G., \& Garmire, G. 1980, MNRAS, 192, 725
Arevalo, M., \& Lazaro, C. 1995, AJ, 110, 1376

Barrado, D., Fernandez-Fugureoa, M. J., Montesinos, B., \& de Castro, E. 1994, A\&A, 290, 137

Borkowski, J. 1988, Internal report of Astronomical Institute in Torun, Poland (in Polish)

Budding, E., \& Zeilik, M. 1987, ApJ, 319, 827

Byrne, P., Eibe, M., \& Rolleston, W. 1996, A\&A, 311, 651

Collier Cameron, A., Duncan, D. K., Ehrehfreund, P., Foing, B. H., Kuntz, K. D., et al. 1990, MNRAS, 247, 415

Djurasevic, G. 1998, A\&AS, 127, 233

Fernandez-Figueroa, M., Montes, D., De Castro, E., \& Cornide, M. 1994, ApJS, 90, 433

Frasca, A., Marino, G., Catalano, S., \& Marilli, E. 2000, A\&A, 358,1007

Frasca, A., \& Catalano, S. 1994, A\&A, 284, 883

Gray, D., in The observations and analysis of stellar photosperes, Cambridge Astrophys. Ser., 20, 248

Hall, J., Huenemoerder, D., Ramsey, L., \& Buzasi, D. 1990, ApJ, 358, 610

Heckert, P., Maloney, G., Steward, G., et al. 1998, AJ, 115, 1145

Hempelmann, A., Hatzes, A., Kurster, M., \& Patkos, L. 1997, A\&A, 317, 125

Herbig, G. 1985, ApJ, 289, 269

Hill, D., Hiditch, R., Younger, F., \& Fisher, W. 1975, MNRAS, 79,131

Hilditch, R., Harland, D., \& McLean, B. 1979, MNRAS, 187, 797

Huisong, T., \& Xuefu, L. 1987, A\&A, 172, 74

Kjurkchieva, D. 1995, ApSS, 232, 185

Kjurkchieva, D., Marchev, D., \& Ogloza, W. 2000a, Acta Astron., 50, 517

Kjurkchieva, D., Marchev, D., \& Ogloza, W. 2000b, A\&A, 354, 909

Kjurkchieva, D., Marchev, D., \& Ogloza, W. 2001, A\&A, 378, 102

Kreiner, J., Kim, C., \& Nha, I. 2001, An atlas of O-C diagrams of eclipsing binary stars (Cracow Pedagogical University Press)

Noyes, R., Hartmann, L., Baliunas, S., Duncan, D. K., \& Vaughan, A. 1984, ApJ, 279, 763

Ozeren, F., Gunn, A., Doyle, J., \& Jevremovic, D. 2001, A\&A, 366,202

Patkos, L. 1982a, Comm. Konkoly Obs., No. 80

Patkos, L. 1982b, in Ejection and Accretion of Matter in Binary Systems, ed. J. Tremko (Bratislava, VEDA), 61

Patkos, L., \& Hempelman, A. 1994, A\&A, 292, 119

Pojmanski, G. 1998, Acta Astron., 48, 711

Popper, D. 1996, ApJS, 106, 133

Rainger, P., Hilditch, R., \& Edwin, R. 1991, MNRAS, 248, 168

Smirnov, O. M., Piskunov, N. E., Afanasyev, V. P., \& Morozov, A. I. 1992, ASP Conf. Ser., 26, Astron. Data Analysis software and Systems, ed. D. Worrall et al., 344

Strassmeier, K., Fekel, F., Bopp, B., Dempsey, R., \& Henri, G. 1990, ApJS, 72, 191

Uchida, Y., \& Sakurai, T. 1983, in Activity in Red Dwarf Stars, ed. P. Byrne, \& M. Rodono, 629

Welty, A., \& Ramsey, L. 1995, AJ, 109, 2187

Zarro, D., \& Rogers, A. 1983, ApJS, 53, 815

Zeilik, M., De Blasi, C., Rhodes, M., \& Budding, E. 1988, ApJ, 332,293 